\title{
Personalizing Thyroid Cancer Care: Are We There Yet?
}

\author{
Naris Nilubol, MD, FACS and Electron Kebebew, MD, FACS \\ Endocrine Oncology Section, Surgery Branch, Center for Cancer Research, National Cancer Institute, Bethesda, MD
}

The prognosis of most well-differentiated thyroid cancers of follicular cells origin is excellent after treatment, but a subset of patients develop locoregional or distant metastases. The overall mortality due to well-differentiated thyroid cancer of follicular cell origin is approximately $1 \%-2 \%$. A number of clinicopathologic prognostic factors such as older age, larger tumor size, extrathyroidal invasion, lymph node and distant metastases, and aggressive tumor histologic subtypes such as tall call, columnar, diffuse sclerosing variants, insular, and Hürthle cell cancer are associated with worse disease-free and overall survival. ${ }^{1}$ Although many prognostic scoring systems have been proposed and accurately predict prognosis, none can be used preoperatively to risk stratify patients and guide surgical treatment. Moreover, subsets of patients with low-risk thyroid cancer develop locoregional and distant metastases.

Our understanding of the molecular events involved in thyroid cancer initiation and progression has improved, and some of these findings have had clinically significant ramifications to the management of patients with thyroid neoplasm. For example, we know now that activating BRAF mutations occur in approximately $50 \%$ of papillary thyroid cancer, the most common type of thyroid cancer. ${ }^{2}$ Activating BRAF mutation is involved in thyroid cancer initiation and progression. The presence of the most common type of BRAF mutation, BRAF V600E, has also been shown to be associated with aggressive disease and worse disease-free survival and documented to improve the diagnostic accuracy of thyroid fine needle aspiration biopsy to diagnose papillary thyroid cancer.,

One other area that has been recently broadly investigated is microRNA (miR) expression and regulation and function in thyroid cancer. MiRs are noncoding, short,

(C) Society of Surgical Oncology 2011

Published Online: 3 May 2011

E. Kebebew, MD, FACS

e-mail: kebebewe@mail.nih.gov single-strand RNAs that regulate post-transcription gene expression. They typically complementarily bind target messenger RNAs and inhibit translation or directly cause messenger RNA degradation. MiRs are important in several biological processes such as cell development, differentiation, and apoptosis. Dysregulated miRs have been identified in numerous cancers, including thyroid cancer. The insight gained from miR biology in cancer can help improve cancer screening and diagnosis, predict tumor response to treatment and prognosis, and identify potential therapeutic targets. ${ }^{5}$

In this issue of the Annals of Surgical Oncology, Yip and colleagues report specific miRs associated with aggressive PTC. The investigators performed miRs expression profiling in 12 frozen papillary thyroid cancer (PTC), the most common type of thyroid cancer, which included 6 aggressive PTC (defined as PTC with local and/ or distant metastasis), 6 nonaggressive PTC, and 4 normal thyroid tissues. MiR 146b, miR-221, miR-222, and miR155 were significantly upregulated in aggressive PTC compared with nonaggressive PTC. MiR-1, miR-34b, miR$130 \mathrm{~b}$, and miR-138 were downregulated in aggressive PTC. The investigators validated their results in 37 additional PTC samples that were matched for clinically variables and the presence of common somatic mutation that occur in PTC (BRAF V600E, K601E, NRAS, HRAS, KRAS). In the validation cohort, they confirmed significant upregulation of miR-146b and miR-222 and downregulation of miR-34b and miR-130 in aggressive PTC. The authors utilized 3 widely used target prediction programs and identified $M E T$, a proto-oncogene that encodes the tyrosine kinase receptor for hepatocyte growth factor, as a predicted target gene of 2 downregulated miR-1 and miR34b. They found higher expression of MET in aggressive PTC. A much higher expression of MET in aggressive PTC with BRAF mutation compared with nonaggressive tumor suggests that this pathway may be independent of BRAF mutation status, although the difference did not reach 
statistically significance. The overexpression of MET described in this study was previously reported in aggressive tall cell variant PTC and PTC with evidence of hypoxia such as tumor necrosis or hemorrhage, psammoma bodies, and cystic degeneration. ${ }^{6,7}$ Scarpino et al. reported the association of increased expression of MET protein and upregulation of hypoxia-induced factor 1 (HIF1) and that the process is more pronounced at tumor periphery. ${ }^{7}$

Yip and colleagues provide additional molecular evidence on how miRs may serve as markers of disease aggressiveness and can target important pathways implicated in carcinogenesis for which there are known agents that inhibit activated pathways such as MET. Certainly, this information can possibly be used to risk stratify patients at the initial evaluation of thyroid nodule(s) and tested in fine needle aspiration biopsy samples, as miRs are relatively stable. This information could be used for initial surgical treatment decisions on the extent of thyroidectomy and need for prophylactic or therapeutic lymph node dissection. Additionally, miR markers of aggressive disease could also be used as an adjunct to routine histologic examination and TNM staging of thyroid cancer samples. Such information could also be used to make treatment decision on the need and dose of $\mathrm{I}^{131}$ ablation, better estimation of the risk of persistent/recurrent disease, and the determination of optimal follow-up. However, before such markers can be used clinically much work needs to be done to validate and demonstrate their clinical utility in large prospective cohort studies across many medical centers. Such studies are necessary for us to implement them in clinical practice, as has been done with molecular testing for BRAF mutation in thyroid FNA biopsy samples to improve diagnostic accuracy and help predict the risk of persistent/recurrent PTC. Unlike mutation analysis, miR expression analysis in clinical samples such as thyroid FNA biopsy or postsurgical tumor tissue represent unique challenges before the clinical application of miR expression as markers of disease aggressiveness. Some of these challenges include the establishment of reference control samples to discern clinically significant differences in miR levels, standardization of miR measurement assays, reproducibility of test results across different laboratories, and prospective clinical studies that can accrue enough patients to demonstrate a difference in disease-free survival and or overall survival. While these are significant challenges that need to be overcome, there has been tremendous progress toward the personalized care of patients with thyroid neoplasm.

\section{REFERENCES}

1. Sipos JA, Mazzaferri EL. Thyroid cancer epidemiology and prognostic variables. Clin Oncol (R Coll Radiol). 2010;22:395404.

2. Wang Y, Ji M, Wang W, Miao Z, Hou P, Chen X, et al. Association of the T1799A BRAF mutation with tumor extrathyroidal invasion, higher peripheral platelet counts, and overexpression of platelet-derived growth factor-B in papillary thyroid cancer. Endocr Relat Cancer. 2008;15:183-90.

3. Xing M, Clark D, Guan H, Ji M, Dackiw A, Carson KA, et al. BRAF mutation testing of thyroid fine-needle aspiration biopsy specimens for preoperative risk stratification in papillary thyroid cancer. J Clin Oncol. 2009;27:2977-82.

4. Shibru D, Chung KW, Kebebew E. Recent developments in the clinical application of thyroid cancer biomarkers. Curr Opin Oncol. 2008;20:13-8.

5. Iorio MV, Croce CM. MicroRNAs in cancer: small molecules with a huge impact. J Clin Oncol. 2009;27:5848-56.

6. Nardone HC, Ziober AF, LiVolsi VA, Mandel SJ, Baloch ZW, Weber RS, et al. c-Met expression in tall cell variant papillary carcinoma of the thyroid. Cancer. 2003;98:1386-93.

7. Scarpino S, Cancellario d'Alena F, Di Napoli A, Pasquini A, Marzullo A, Ruco LP. Increased expression of Met protein is associated with up-regulation of hypoxia inducible factor-1 (HIF1 ) in tumour cells in papillary carcinoma of the thyroid. $J$ Pathol. 2004;202:352-8. 